\title{
Stress Transfer Modeling in Viscoelastic Polymer Matrix Composites
}

\author{
Biju J. Thuruthimattam*and Anthony M. Waas ${ }^{\dagger}$ \\ Composite Structures Laboratory \\ Department of Aerospace Engineering \\ University of Michigan, Ann Arbor, MI 48109. \\ Alan S. Wineman ${ }^{\ddagger}$ \\ Department of Mechanical Engineering \\ University of Michigan, Ann Arbor, MI 48109.
}

\begin{abstract}
A viscoelastic analysis of a single fiber unidirectional composite model is carried out to investigate the time-dependent stress transfer between an elastic fiber and a viscoelastic matrix. The stress state in the composite is solved at incremental time intervals to obtain a stabilized solution, and the time required to reach stability. The results obtained from a linear viscoelastic analysis is compared with the corresponding result obtained by an elastic analysis of the fiber and the matrix. It is found that there is a significant decrease in the composite axial modulus, which takes place over a large period of time.
\end{abstract}

\section{Introduction}

It is well established that the mechanical behavior of typical polymers that are used as the matrix in fiber reinforced polymer matrix composites (PMC) is time-dependent. Thus, one would expect the properties of a PMC to also be time-dependent, and over a period of time comparable to that required for the relaxation of the matrix. In polymer matrix composites, the fraction of the load carried by the matrix in on-axis loading is usually quite small as compared to that carried by the fiber, due to the relatively large mismatch in axial stiffness. A change in the elastic properties of the matrix is not expected

\footnotetext{
* Ph. D candidate

†Associate Professor of Aerospace Engineering. Associate Fellow AIAA.

${ }^{\ddagger}$ Professor of Mechanical Engineering and Applied Mechanics

${ }^{0}$ Copyright (C) 1999 by Biju Thuruthimattam. Published by the American Institute of Aeronautics and Astronautics, Inc. by permission.
}

to make significant differences to the load carrying properties of the composite. This is one of the reasons why the time-dependent elastic properties of the matrix are usually neglected in traditional modeling of PMCs when subjected to predominantly onaxis constant strain loadings. Over a period of time, the properties of the matrix in the composite can relax, due to which the mechanics of stress-transfer between the fiber and the matrix changes.

The stress transfer from the matrix to a fiber was considered by Cox [1], who used a concentric cylinder of matrix that contained a centrally located discontinuous fiber. An elastic analysis was carried out by Cox, who found that a minimum fiber length was necessary in order to achieve the maximum possible stiffness for a given matrix/fiber combination. When the matrix is time-dependent, it becomes necessary to re-examine the results obtained by Cox, with respect to issues pertaining to the change in the mechanics of stress transfer from the matrix to the fiber. In particular, the time required for the stress distribution to reach a stable state and the effect of the matrix and fiber properties in influencing this time need to be characterized.

Using the assumptions of linear viscoelasticity to model the matrix, the final stress state of the matrix can be obtained, and this can easily shown to be consistent with the Cox type elastic analysis carried out with the matrix properties replaced by the large time elastic properties of the matrix. However, the time for stabilization (i.e. the time required for the fiber and matrix stress to reach a stable state) cannot be predicted in a similar manner, nor the variation of this parameter with respect to the properties of the matrix.

The goal of this study then is to investigate 
the time dependent mechanics of stress transfer during interaction between a strained matrix and an embedded fiber, as a function of the matrix timedependent properties, where the matrix is modeled as a viscoelastic solid. The loading envisioned in this study is a constant strain loading. A transient viscoelastic analysis is carried out to obtain parameters for comparison with the elastic analysis using the fiber and initial matrix properties. In these analyses, the parameters of interest are the fiber half-length, the stress-transfer length and the maximum stress arising on the fiber. If the fiber half-length is larger than a minimum required value, then complete stress buildup occurs over the stress transfer length, and there is no decrease in the maximum stress.

\section{Modeling}

Consider a concentric cylinder of fiber-matrix with an embedded fiber of length $2 L$ as shown in Figure 1. With respect to the coordinate axes as denoted in the figure, the radial variation of shear stress in the matrix, $\tau$ ( at a given axial distance from the fiber midpoint ) is obtained by equating the shear forces on neighboring annuli, with radii $z_{1}$ and $z_{2}$ of length $d x$.

$$
\begin{gathered}
2 \pi z_{1} \tau_{1} d x=2 \pi z_{2} \tau_{2} d x \\
\text { i.e. } \frac{\tau_{1}}{\tau_{2}}=\frac{z_{2}}{z_{1}}
\end{gathered}
$$

Hence, at any radius $z$, the shear stress $\tau(z)$ can be related to the interface shear stress $\tau_{i}$ as

$$
\tau(z)=\tau_{i}\left(\frac{r_{i}}{z}\right)
$$

where $r_{i}$ is the radial distance of the interface from the center of the fiber.
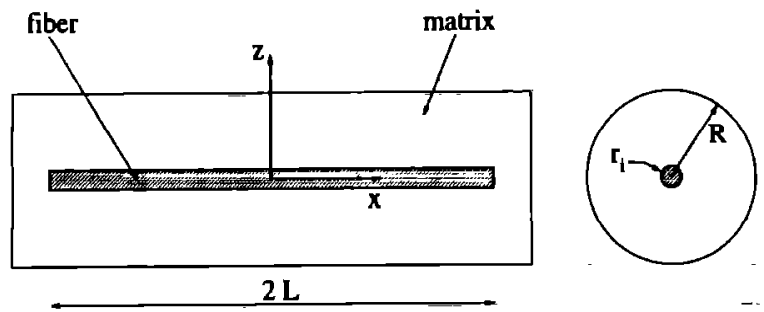

Figure 1: Single fiber composite model.

When the matrix considered is modeled as a viscoelastic solid, the stress becomes a function of time and radial distance. For a viscoelastic matrix, it is assumed that time dependent processes are quasi-static, so that inertia can be ignored. Equation (1) can then be rewritten as

$$
\tau_{m}(z, t)=\frac{r_{i}}{z} \tau_{i}(t)
$$

where, a subscript $m$ stands for matrix and $i$ stands for interface.

Consider the axial displacement $u_{x}(x, z, t)$ of the matrix. In order to develop the axial equilibrium equation for the fiber, we shall use a reduce notation for the matrix displacement, so that $u_{x}(z, t)$ is understood to represent the axial displacement at a given $x$ location. Then,

$$
\begin{aligned}
\frac{\partial u(z, t)}{\partial z} & =\int_{0^{-}}^{t} J_{m}(t-s) d\left(\tau_{m}(z, s)\right) \\
& =\int_{0^{-}}^{t} J_{m}(t-s) d\left(\frac{r_{i}}{z} \tau_{i}(s)\right) \\
& =\frac{r_{i}}{z} \int_{0^{-}}^{t} J_{m}(t-s) d\left(\tau_{i}(s)\right) \\
& =\frac{r_{i}}{z} J_{m} * d \tau_{i}(t),
\end{aligned}
$$

where, $J_{m}(t)$ is the time dependent shear creep compliance of the matrix, and where the notation $J_{m} * d \tau_{i}$ is used to represent the integral expression. Integrating in the radial direction,

$$
u_{m}(R, t)-u_{m}\left(r_{i}, t\right)=r_{i} \ln \left(\frac{R}{r_{i}}\right) J_{m} * d \tau_{i}(t)
$$

where at time $t, u_{m}(R, t)$ represents the matrix displacement at $z=R$, and $u_{m}\left(r_{i}, t\right)$ represents the matrix displacement at the interface. $R$ is chosen remote from the vicinity of the fiber such that at that location, the matrix strain is uniform. The approximate value of $R$ is dictated by the proximity of the neighboring fibers, and hence the fiber arrangement and the fiber volume fraction $V_{f}$. For a hexagonal array,

$$
\left(\frac{R}{r_{i}}\right)^{2}=\frac{\pi}{2 V_{f} \sqrt{3}} \approx \frac{0.9}{V_{f}}
$$

Assuming a perfect bond between fiber and matrix, $u\left(r_{i}, t\right)=u_{f}(t)$, where $u_{f}(t)$ is the fiber axial displacement and

$u_{m}(R, t)-u_{f}(t)=r_{i} J_{m} * d \tau_{i}(t) \ln \left(\frac{R}{r_{i}}\right)$

Thus,

$$
\frac{u_{m}(R, t)-u_{f}(t)}{r \ln \left(\frac{R}{r_{i}}\right)}=J_{m} * d \tau_{i}
$$

Hence, the interfacial shear stress can be obtained as

$$
\tau_{i}(t)=\frac{1}{r_{i} \ln \left(\frac{R}{r_{i}}\right)}\left(u_{m}(R, t)-u_{f}(t)\right) * d G_{m},
$$


where, $G_{m}(t)$ is the shear stress relaxation function for the matrix. For most PMCs, the axial modulus of the fiber is much higher than the corresponding extensional modulus of the matrix. Hence, the axial stress in the matrix is much smaller than that in the fiber and can be ignored. For the polymer matrix material, the maximum value of the stress relaxation function of the matrix is sufficiently small that this assumption is still reasonable. Noting this, from the force equilibrium of an element of the fiber, where the fiber is modeled as a one-dimensional linear elastic solid,

$$
2 \pi r_{i} \tau_{i} d x=-\pi r_{i}^{2} d \sigma_{f}
$$

and hence, assuming perfect adhesion and no shear strain in the fiber,

$$
\frac{\partial \sigma_{f}}{\partial x}=-\frac{2 \tau_{i}}{r_{i}}
$$

Using the axial equilibrium of the fiber as given in Equation (7),

$$
\frac{\partial \sigma_{f}}{\partial x}=-\frac{2}{r_{i}}\left(\frac{1}{r_{i} \ln \left(\frac{R}{r_{i}}\right)}\right)\left(u_{m}(R, t)-u_{f}(t)\right) * d G_{m}
$$

Differentiating with respect to $x$,

$$
\begin{aligned}
\frac{\partial^{2} \sigma_{f}}{\partial x^{2}} & =-\frac{2}{r_{i}^{2}} \frac{1}{\ln \left(\frac{R}{r_{i}}\right)}\left(\epsilon_{m}-\frac{\partial u_{f}}{\partial x}\right) * d G_{m} \\
& =-\frac{2}{r_{i}^{2}} \frac{1}{\ln \left(\frac{R}{r_{i}}\right)}\left(\epsilon_{m}-\frac{\sigma_{f}}{E_{f}}\right) * d G_{m}
\end{aligned}
$$

where $\epsilon_{m}$ denotes axial stresses in the matrix at radius $R, \frac{\partial u_{f}}{\partial x}$ is the axial strain in the fiber, and $E_{f}$ is the axial modulus of the fiber. Rearranging the above equation,

$\frac{\partial^{2} \sigma_{f}}{\partial x^{2}}-\frac{2}{r_{i}^{2} E_{f}} \frac{1}{\ln \left(\frac{R}{r_{i}}\right)} \sigma_{f} * d G_{m}=-\frac{2}{r_{i}^{2}} \frac{1}{\ln \left(\frac{R}{r_{i}}\right)} \epsilon_{m} * d G_{m}$

The tensile stress in the linear elastic fiber is then governed by the differential-Volterra integral equation,

$$
\begin{gathered}
\frac{\partial^{2} \sigma_{f}(x, t)}{\partial x^{2}}-\frac{2}{r_{i}^{2} E_{f}} \cdot \frac{1}{\ln \left(\frac{R}{r_{i}}\right)}\left[\sigma_{f}(x, t) G_{m}(0)+\right. \\
\left.\int_{0}^{t} \sigma_{f}(x, s) \dot{G}_{m}(t-s) d s\right]=-\frac{2}{r_{i}^{2}} \cdot \frac{1}{\ln \left(\frac{R}{r_{i}}\right)} \epsilon_{m} * d G_{m}
\end{gathered}
$$

We parameterize equation (10) using the following parameter:

$$
\beta_{0}^{2}=\frac{2 G_{m}(0)}{E_{f} \ln \left(\frac{R}{r_{i}}\right)}
$$

Hence equation (10) simplifies as

$$
\begin{aligned}
& \frac{\partial^{2} \sigma_{f}(x, t)}{\partial x^{2}}-\left(\frac{\beta_{0}}{r_{i}}\right)^{2}\left[\sigma_{f}(x, t)+\right. \\
& \left.\frac{1}{G_{m}(0)} \int_{0}^{t} \sigma_{f}(x, s) \dot{G}_{m}(t-s) d s\right] \\
& =-\left(\frac{\beta_{0}}{r_{i}}\right)^{2}\left(\frac{E_{f}}{G_{m}(0)}\right) \epsilon_{m} d G_{m}
\end{aligned}
$$

For a step strain,

$$
\epsilon_{m} * d G_{m}=\int_{0^{+}}^{t} \epsilon_{m}(t-s) d G_{m}(s)=\epsilon_{0} G_{m}(t)
$$

In order to bring out the essential features of viscoelastic response, it is assumed that

$$
G_{m}(t)=G_{m}(\infty)+\left(G_{m}(0)-G_{m}(\infty)\right) e^{-\left(t / \tau_{m}\right)},
$$

where, $G_{m}(0)$ represents the instantaneous modulus, $G_{m}(\infty)$ represents the large time equilibrium modulus and $\tau$ is the characteristic relaxation time for the epoxy matrix.

In a polymer matrix undergoing relaxation, all the elastic properties change with time. However, the effect of a change in the Poisson's ratio not being significant, it is assumed to be constant for the purposes of modeling. The time dependent Young's Modulus, $E_{m}$ of the isotropic matrix is related to the shear modulus as,

$$
E_{m}(t)=2\left(1+\nu_{m}\right) \cdot G_{m}(t)
$$

and this expression is used later to compute the composite time dependent modulus.

\subsection{Analytical solution at time $t=0$}

The initial conditions for the solution of the differential equation are obtained by solving the above equation analytically at time $t=0$. We obtain the following ordinary differential equation

$$
\frac{d^{2} \sigma_{f}(x, 0)}{d x^{2}}-\left(\frac{\beta_{0}}{r_{i}}\right)^{2} \sigma_{f}(x, 0)=-\left(\frac{\beta_{0}}{r_{i}}\right)^{2} E_{f} \epsilon_{0}
$$

We note that the system is symmetrical and assume that there is no stress on the end of the fiber, similar to that assumed by Cox. We also introduce the following non-dimensional length parameter 


$$
s=\frac{L}{r_{i}}
$$

The boundary conditions are given as

$$
\begin{aligned}
& \frac{\partial \sigma_{f}(0, t)}{\partial x}=-0 \\
& \sigma_{f}(L, t)=0
\end{aligned}
$$

Solving equation (13), we get the solution

$$
\sigma_{f}(x, 0)=E_{f} \epsilon_{0}\left[1-\frac{\cosh \left(\beta_{0}\left(x / r_{i}\right)\right)}{\cosh \left(\beta_{0} s\right)}\right]
$$

The average stress over the fiber length can then be defined as

$$
\bar{\sigma}_{f}(0)=\frac{1}{L} \int_{0}^{L} \sigma_{f}(x, 0) d x
$$

For axial force equilibrium of the composite,

$$
A_{f} \bar{\sigma}_{f}(0)+A_{m} E_{m}(0) \epsilon_{0}=A_{c} E_{c} \epsilon_{0},
$$

where a subscript ' $c$ ' is used to denote composite properties, and $A_{c}=A_{f}+A_{m}$. It must be noted that the shear relaxation time is generally different than the extensional relaxation time for an isotropic solid. However, since we are assuming that the poissons ration is time-independent in the present work, the extensional relaxation function $E_{m}(t)$ is obtained as stated earlier.

Thus,

$$
E_{c}(0)=V_{f} \frac{\bar{\sigma}_{f}(0)}{\epsilon_{0}}+\left(1-V_{f}\right) E_{m}(0),
$$

where, $V_{f}=\frac{A_{m}}{A_{c}}$. Thus, the equivalent longitudinal Young's modulus of the composite at time $t=0$ can be obtained.

\subsection{Analytical solution at time $t=\infty$}

For large time, the system will have stabilize to a steady stress distribution, $\sigma_{f}(x, \infty)$. The convolution integral in equation (10) will have converged to $\sigma_{f}(x, \infty) G_{m}(\infty)$, and the following governing equation will be obtained;

$$
\begin{gathered}
\frac{\partial^{2} \sigma_{f}(x)}{\partial x^{2}}-\left(\frac{\beta_{0}}{r_{i}}\right)^{2} \sigma_{f}(x)\left[\frac{G_{m}(\infty)}{G_{m}(0)}\right] \\
-\left(\frac{\beta_{0}}{r_{i}}\right)^{2}\left(\frac{E_{f}}{G_{m}(0)}\right) \epsilon_{0} G_{m}(\infty)
\end{gathered}
$$

This can be reduced to

$$
\frac{d^{2} \sigma_{f}(x)}{d x^{2}}-\left(\frac{\beta_{\infty}}{r_{i}}\right)^{2} \sigma_{f}(x)=-\left(\frac{\beta_{\infty}}{r_{i}}\right)^{2} E_{f} \epsilon_{0}
$$

where,

$$
\beta_{\infty}^{2}=\frac{2 G_{m}(\infty)}{E_{f} \ln \left(\frac{R}{r_{i}}\right)}
$$

The boundary conditions are independent of time, and hence will also apply for the above equation. Hence the stress distribution for large time is,

$$
\sigma_{f}(x, \infty)=E_{f} \epsilon_{0}\left[1-\frac{\cosh \left(\beta_{\infty}\left(x / r_{i}\right)\right)}{\cosh \left(\beta_{\infty} s\right)}\right]
$$

The average stress and the composite longitudinal Young's modulus for large time can be defined as

$$
\begin{aligned}
& \bar{\sigma}_{f}(\infty)=\frac{1}{L} \int_{0}^{L} \sigma_{f}(x, \infty) d x \\
& E_{c}(\infty)=V_{f} \frac{\bar{\sigma}_{f}(\infty)}{\epsilon_{0}}+\left(1-V_{f}\right) E_{m}(\infty)
\end{aligned}
$$

\section{Transient Solution of the Linear Viscoelastic Problem}

The above arguments provide properties of the composite at the two end times $t=0$ and $t=\infty$, but provide no useful information about the time required for the composite stress state to stabilize. Hence, a transient solution of the governing equation is required. The transient solution is obtained using the method indicated below.

To solve for the fiber stresses at any time $t=t_{p}$, we use a numerical approximation to equation (10), writing the differential term as a difference expression and the integral term as a discrete sum using the trapezoidal rule

$$
\begin{aligned}
& \left(\frac{\sigma_{n-1 p}-2 \sigma_{n p}+\sigma_{n+1 p}}{\Delta x^{2}}\right) \\
& \quad-\left(\frac{\beta_{0}}{r_{i}}\right)^{2}\left[\sigma_{n p}+\frac{1}{G_{m}(0)}\left(\frac{1}{2} \sigma_{n 0} \dot{G}_{m}\left(t_{p}\right) \Delta t\right.\right. \\
& \left.\left.\quad+\sum_{k=1}^{p-1} \sigma_{n k} \dot{G}_{m}\left(t_{p}-k \Delta t\right) \Delta t+\frac{1}{2} \sigma_{n p} \dot{G}_{m}(0) \Delta t\right)\right] \\
& =-\left(\frac{\beta_{0}}{r_{i}}\right)^{2}\left(\frac{E_{f}}{G_{m}(0)}\right) \epsilon_{0} G_{m}\left(t_{p}\right)
\end{aligned}
$$

This is written as a system of linear simultaneous equations in terms of the stresses at discrete 
points along the fiber, with $n=1, \ldots, N+1$ representing the points along the fiber length, from $x=0$, to $x=L$. The sum $k=0, \ldots, p$ is used to indicate a summation of the stresses at a single point at different time steps. The equations used to solve for the distributed point stresses is given in (20), on the next page.

The boundary conditions are implemented in terms of the stresses at discrete points as

$$
\begin{array}{cc}
\sigma_{1 p}=\sigma_{2 p} & \text { for all } p \\
\sigma_{N+1 p}=0 & \text { for all } p
\end{array}
$$

where the fiber is discretized in space from $x=$ 0 (corresponding to $n=1$ ) to $x=L$ (corresponding to $n=N=1$ ), and the above equation is evaluated for $n=2$ to $n=N$. Note that the system matrix has dimension $(N-1) x(N+1)$ and is not square. Let $K_{1}, K_{2}$ and $\alpha$ be defined as

$$
\begin{aligned}
K_{1} & =\left(\frac{-1}{\Delta x^{2}}\right)-\left(\frac{\beta_{0}}{r_{i}}\right)^{2}-\left(\frac{1}{2}\right)\left(\frac{\beta_{0}}{r_{i}}\right)^{2}\left[\frac{\dot{G}_{m}(0) \Delta t}{G_{m}(0)}\right] \\
K_{2} & =\left(\frac{-2}{\Delta x^{2}}\right)-\left(\frac{\beta_{0}}{r_{i}}\right)^{2}-\left(\frac{1}{2}\right)\left(\frac{\beta_{0}}{r_{i}}\right)^{2}\left[\frac{\dot{G}_{m}(0) \Delta t}{G_{m}(0)}\right] \\
\alpha & =\frac{1}{\Delta x^{2}}
\end{aligned}
$$

In the limiting case, as the finite time step becomes infinitesimal, the expressions for $K_{1}$ and $K_{2}$ tend to

$$
\begin{aligned}
& K_{1} \approx\left(\frac{-1}{\Delta x^{2}}\right)-\left(\frac{\beta_{0}}{r_{i}}\right)^{2} \\
& K_{2} \approx\left(\frac{-2}{\Delta x^{2}}\right)-\left(\frac{\beta_{0}}{r_{i}}\right)^{2}
\end{aligned}
$$

The terms relating to the stresses at time $t_{p}$ are collected to form the coefficient matrix for a system of simultaneous linear algebraic equations as shown in Equation (21), on the next page. i.e.

$$
[D]\left\{\sigma_{t_{p}}\right\}=\left\{R_{t_{p}-\Delta t}\right\}
$$

Thus we can obtain the stress distribution along the length of the fiber at any time using the stress distributions at all previous time instants as

$$
\left\{\sigma_{t_{p}}\right\}=[D]^{-1}\left\{R_{t_{p}-\Delta t}\right\}
$$

The verification of the transient model is easily carried out by comparing the average stress, the maximum stress and the stress transfer length, after the system has stabilized, with the analytical predictions. The stress transfer length was defined as that length over which the fiber stress builds up to $99 \%$ of the maximum fiber stress at a given instant of time. Recall that the fiber stress is zero at the free end $(x= \pm L)$, and builds up gradually to a maximum which is reached at $x=0$. The manner in which this buildup occurs is given by the expression for the fiber stress, $\sigma_{f}(x, t)$. For example, the previous analytical solutions show the exponential character of this buildup at $t=0$ and for large times (see the expressions for $\sigma_{f}(x, 0)$ and $\sigma_{f}(x, \infty)$ obtained earlier).

From the transient modeling, the fiber stress distribution, the average fiber stress, and the longitudinal Young's modulus of the composite can be obtained as functions of time.

\section{Results of the Transient Vis- coelastic Analysis}

Studies were carried out for the material properties given in Table 1. Values of static parameters used in the linear model are indicated in Table 2. Different relaxation ratios $\left(G_{m}(0) / G_{m}(\infty)\right)$ were considered, in order to compare responses of different possible material systems.

The results obtained from the analytical modeling are given in Tables 3 and 4. These tables display results obtained from linear elastic analyses, with the appropriate matrix properties replaced by the instantaneous values of matrix properties at time $t=0$ and $t=\infty$. As the relaxation ratios become large, these analyses show that the minimum embedded fiber half-length $L_{\min }$, required for complete stress buildup also increases. Thus, for a fixed embedded half-length $L$, there is a maximum relaxation ratio, beyond which complete stress build up will not occur. For example, Tables 3 and 4 were constructed for E-glass/epoxy and Carbon/epoxy respectively, with a fixed $L / r=3000$. With respect to Table 3, when $G_{m}(0) / G_{m}(\infty)=2000, l_{\infty} / r=790$ while $L_{m i n} / r=2473$, but for $G_{m}(0) / G_{m}(\infty)=3175$, $l_{\infty} / r=989$ and $L_{\min } / r=3115>3000$ ! Thus for E-glass/epoxy and for $L / r=3000$, complete stress buildup will not occur for relaxation ratios exceeding beyond approximately 3000 . The corresponding relaxation ratio for Carbon/epoxy, from Table 4, is about 600 .

These analytical predictions are useful for the proceeding linear viscoelastic analyses, however, a complete numerical solution of the system equations is still needed to obtain the time required for the 


$$
\begin{gathered}
\frac{1}{\Delta x^{2}}\left[\begin{array}{cccccccc}
1 & -2 & 1 & 0 & \cdots & 0 & 0 & 0 \\
0 & 1 & -2 & 1 & 0 & \cdots & 0 & 0 \\
0 & 0 & 1 & -2 & 1 & 0 & \cdots & 0 \\
& \vdots & & \ddots & \ddots & \ddots & & \vdots \\
0 & 0 & 0 & \cdots & 1 & -2 & 1 & 0 \\
0 & 0 & 0 & 0 & \cdots & 1 & -2 & 1
\end{array}\right]\left\{\begin{array}{c}
\sigma_{1 p} \\
\sigma_{2 p} \\
\sigma_{3 p} \\
\vdots \\
\sigma_{N p} \\
\sigma_{N+1}
\end{array}\right\}-\left(\frac{\beta}{r_{i}}\right)^{2}\left\{\begin{array}{c}
\sigma_{2 p} \\
\sigma_{3 p} \\
\vdots \\
\sigma_{N p}
\end{array}\right\} \\
-\left(\frac{\beta}{r_{i}}\right)^{2} \frac{1}{G_{m}(0)}\left\{\begin{array}{c}
\frac{1}{2} \sigma_{20} \dot{G}_{m}\left(t_{p}\right) \Delta t+\sum_{k=1}^{p-1} \sigma_{2 k} \dot{G}_{m}^{k}\left(t_{p}-k \Delta t\right) \Delta t+\frac{1}{2} \sigma_{2 p} \dot{G}_{m}(0) \Delta t \\
\frac{1}{2} \sigma_{30} \dot{G}_{m}\left(t_{p}\right) \Delta t+\sum_{k=1}^{p=1} \sigma_{3 k} \dot{G}_{m}^{k}\left(t_{p}-k \Delta t\right) \Delta t+\frac{1}{2} \sigma_{3 p} \dot{G}_{m}(0) \Delta t \\
\vdots \\
\frac{1}{2} \sigma_{N 0} \dot{G}_{m}\left(t_{p}\right) \Delta t+\sum_{k=1}^{p-1} \sigma_{N k} \dot{G}_{m}^{k}\left(t_{p}-k \Delta t\right) \Delta t+\frac{1}{2} \sigma_{N p} \dot{G}_{m}(0) \Delta t
\end{array}\right\} \\
=-\left(\frac{\beta}{r_{i}}\right)^{2}\left(\frac{E_{f} \epsilon_{0} G_{m}\left(t_{p}\right)}{G_{m}(0)}\right)\left\{\begin{array}{c}
1 \\
1 \\
\vdots \\
1
\end{array}\right\}
\end{gathered}
$$

$$
\begin{aligned}
& {\left[\begin{array}{cccccc}
K_{1} & \alpha & 0 & \cdots & 0 & 0 \\
\alpha & K_{2} & \alpha & 0 & \cdots & 0 \\
0 & \alpha & K_{2} & \alpha & 0 & \cdots \\
\vdots & \ddots & \ddots & \ddots & \ddots & \ddots \\
0 & \cdots & 0 & \alpha & K_{2} & \alpha \\
0 & 0 & \cdots & 0 & \alpha & K_{2}
\end{array}\right]\left\{\begin{array}{c}
\sigma_{2 p} \\
\sigma_{3 p} \\
\sigma_{4 p} \\
\vdots \\
\sigma_{N-1 p} \\
\sigma_{N p}
\end{array}\right\}} \\
& =\left(\frac{\beta}{r_{i}}\right)^{2}\left(\frac{1}{G_{m}(0)}\right)\left\{\begin{array}{cc}
\frac{1}{2} \sigma_{20} \dot{G}_{m}\left(t_{p}\right) \Delta t+\sum_{k=1}^{p-1} \sigma_{2 k} \dot{G}_{m}^{k}\left(t_{p}-k \Delta t\right) \Delta t & -E_{f} \epsilon_{0} G_{m}\left(t_{p}\right) \\
\frac{1}{2} \sigma_{30} \dot{G}_{m}\left(t_{p}\right) \Delta t+\sum_{k=1}^{p=1} \sigma_{3 k} \dot{G}_{m}^{k}\left(t_{p}-k \Delta t\right) \Delta t & -E_{f} \epsilon_{0} G_{m}\left(t_{p}\right) \\
\frac{1}{2} \sigma_{40} \dot{G}_{m}\left(t_{p}\right) \Delta t+\sum_{k=1}^{p=1} \sigma_{4 k} \dot{G}_{m}^{k}\left(t_{p}-k \Delta t\right) \Delta t & -E_{f} \epsilon_{0} G_{m}\left(t_{p}\right) \\
\vdots & \\
\frac{1}{2} \sigma_{N-10} \dot{G}_{m}\left(t_{p}\right) \Delta t+\sum_{k=1}^{p-1} \sigma_{N-1 k} \dot{G}_{m}^{k}\left(t_{p}-k \Delta t\right) \Delta t & -E_{f} \epsilon_{0} G_{m}\left(t_{p}\right) \\
\frac{1}{2} \sigma_{N 0} \dot{G}_{m}\left(t_{p}\right) \Delta t+\sum_{k=1}^{p-1} \sigma_{N k} \dot{G}_{m}^{k}\left(t_{p}-k \Delta t\right) \Delta t & -E_{f} \epsilon_{0} G_{m}\left(t_{p}\right)
\end{array}\right\}
\end{aligned}
$$

stress state to stabilize. Using the previous analytical predictions, full numerical solutions of the linear viscoelastic models were carried out for the $\mathrm{E}$ glass/epoxy and Carbon/epoxy systems considered previously. Relaxation ratios in the range 5-3175 for E-glass/epoxy and 5-2000 for Carbon/epoxy were considered. The time required for the stress to stabilize and the composite final modulus were computed and these are listed in Tables 6 and 7 respectively. In Table 6, in general, note that the time required for the system to reach a stable state is much larger than the time required for the matrix to reach its final degraded value. In other words, the system behavior tends to possess a 'memory', in that its own time constant to reach a stable state is markedly different from what can be inferred by examining the matrix time-dependent behavior by itself. This holds true for both E-glass/epoxy (Table 5) and Carbon/epoxy (Table 6).

During the transient time period, Figure 2 shows the axial stress distribution predicted using the linear viscoelastic model, for a Carbon/epoxy composite, with $G_{m}(0) / G_{m}(\infty)=1000$ at time $t=$ 0 (elastic) and time $t=400 \mathrm{sec}$. The viscoelastic model predicts that there is a significant change in the stress distribution near the free ends of the fibcr. These differences in prediction can plan a crucial role in explaining fiber end failure, as well as aid in understanding how stress relaxation effects influence the mechanics of stress transfer from the matrix to the fiber.

Figure 3 shows a plot of the time-dependent composite axial modulus, $E_{c}(t)$ for a Carbon/epoxy composite, based on the linear viscoelastic models. 
In conjunction with the second column of Table 6 , it is evident that the composite time-dependent modulus does not show the same trend as assumed for the matrix modulus time-dependency. In other words, the combination of a time-independent elastic fiber and a time-dependent matrix leads to a complex interaction which affects the composite properties in a manner that can only be deduced by solving the complete boundary/initial value problem as has been described herein. In general, the system time constant is always larger, often by orders of magnitude. Further work at exploring the use of a nonlinear viscoelastic model for the composite is presently being carried out [2].

\section{Conclusions}

The analysis of a elastic fiber in a viscoelastic matrix has becn carricd out in this paper. Analytically obtainable results are indicated, which are then used for verification of the linear analyses. The properties of the matrix are considered to be linearly viscoelastic. The solution of the governing equation leads to the results indicated in Tables 5 and 6 . As the relaxation ratio increases, the stress transfer length increases quite significantly. The stress transfer length is found to tend to a constant value for the higher relaxation ratios considered in this study. This is because as the relaxation ratio increases one needs a increasing length of embedded fiber to achieve complete stress transfer. If the embedded length is insufficient for this purpose incomplete stress transfer occurs and the length over which this transfer takes place is equal to the embedded fiber half length regardless of the fiber length that is actually required. In this study, the embedded half length was fixed at $L / r=3000$. The time required for the stress state to stabilize also increases as the relaxation ratio increases, often by orders of magnitude.

\section{Acknowledgments}

This work was supported by the François-Xavier Bagnoud (FXB) Association.

\section{References}

1. Cox, H.L., British Journal of Applied Physics, 1952, Vol. 3, pp. 72.

2. Thuruthimattam, B.J., Waas, A.M. and Wineman, A.S., "The Effect of a Nonlinear Viscoelastic Clock Model on the Stress Transfer in a
Fiber-reinforced Polymer Matrix Composite", in preparation, 1999. 
Table 1: Elastic Properties of fiber and matrix.

\begin{tabular}{lc}
\hline \hline Properties & Value \\
\hline \hline Young's Modulus of E-glass fiber $E_{f}$ & $72.4 \mathrm{GPa}$ \\
Young's Modulus of Carbon fiber, $E_{f}$ & $365 \mathrm{GPa}$ \\
Young's Modulus of Matrix, $E_{m}$ & $3.5 \mathrm{GPa}$ \\
Initial Shear Modulus of Poly-Vinyl Acetate matrix, $G_{m}(0)$ & $635 \mathrm{MPa}$ \\
Poisson's Ratio of matrix $\nu_{m}$ & 0.3 \\
Characteristic Time Period of matrix, $\tau_{m}$ & $1 \mathrm{sec}$ \\
\hline \hline
\end{tabular}

Table 2: Values of Static Parameters.

\begin{tabular}{lc}
\hline \hline Parameter & Value \\
\hline \hline $\mathrm{V}_{f}$ (Volume fraction) & 0.49 \\
$\epsilon_{0}$ (Initial Strain) & 0.001 \\
$r$ (Radius of fiber) & 10 microns \\
$\tau$ (Time Constant of $G_{m}$ ) & $1 \mathrm{sec}$ \\
$\mathrm{L}$ (Half-length of fiber) & $30 \mathrm{~mm}$ \\
\hline \hline
\end{tabular}


Table 3: Stress Transfer Lengths and Fiber lengths: Final Stabilized length $\left(l_{\infty} / r\right)$ and Minimum Fiber half-length $\left(L_{\min } / r\right)$ required for E-Glass/epoxy, $L / r=3000, l_{0} / \tau=18$.

\begin{tabular}{ccc}
\hline \hline $\mathrm{G}_{0} / \mathrm{G}_{\infty}$ & $l_{\infty 0} / r$ & $L_{\min } / r$ \\
\hline \hline 5 & 40 & 124 \\
10 & 60 & 175 \\
20 & 80 & 248 \\
50 & 300 & 391 \\
100 & 180 & 553 \\
200 & 250 & 782 \\
500 & 400 & 1237 \\
1000 & 555 & 1749 \\
2000 & 790 & 2473 \\
3175 & 989 & 3115 \\
5000 & 1240 & 3909 \\
10000 & 1728 & 5528 \\
\hline \hline
\end{tabular}

Table 4: Stress Transfer Lengths and Fiber lengths: Final Stabilized length $\left(l_{\infty} / r\right)$ and Minimum Fiber half-length $\left(L_{\min } / r\right)$ required for Carbon/epoxy, $L / r=3000, l_{0} / r=40$.

\begin{tabular}{ccc}
\hline \hline $\mathrm{G}_{0} / \mathrm{G}_{\infty}$ & $l_{\infty} / r$ & $L_{\min } / r$ \\
\hline 5 & 89 & 278 \\
10 & 125 & 393 \\
20 & 177 & 556 \\
50 & 279 & 878 \\
100 & 400 & 1242 \\
200 & 558 & 1756 \\
500 & 881 & 2776 \\
1000 & 1246 & 3925 \\
2000 & 1734 & 5551 \\
3175 & 2071 & 6994 \\
5000 & 2320 & 8777 \\
10000 & 2527 & 12412 \\
\hline
\end{tabular}


Table 5: Results of Viscoelastic Analysis - E-Glass-epoxy $(L / r=3000)$

\begin{tabular}{|c|c|c|c|c|c|c|}
\hline $\mathrm{G}_{0} / \mathrm{G}_{\infty}$ & $\mathrm{t}_{\infty}^{\mathrm{m}}$ & & $t_{\infty}^{l}$ & $\sigma_{f}(\infty)(\mathrm{MPa})$ & $\mathrm{E}_{c}(\infty)(\mathrm{GPa})$ & $\Delta E_{c}(\%)$ \\
\hline 5 & 5.9915 & & 2.54 & 72.19 & 35.72 & 3.96 \\
\hline 10 & 6.8024 & & 6.72 & 72.11 & 35.50 & 4.54 \\
\hline 20 & 7.5496 & & 15.76 & 71.99 & 35.36 & 4.93 \\
\hline 50 & 8.4970 & - & 155.72 & 71.75 & 35.19 & 5.39 \\
\hline 100 & 9.2003 & - & 242.68 & 71.48 & 35.04 & 5.79 \\
\hline 200 & 9.8985 & & 761.52 & 71.10 & 34.85 & 6.31 \\
\hline 500 & $=10.8178$ & & 2471.04 & 70.34 & 34.47 & 7.32 \\
\hline 1000 & 11.5119 & - & 3950.00 & 69.49 & 34.05 & 8.44 \\
\hline 2000 & 12.2056 & & 8878.06 & 68.29 & 33.46 & 10.03 \\
\hline 3175 & 12.6679 & & 15292.10 & 67.22 & 32.95 & 11.44 \\
\hline
\end{tabular}

Table 6: Results of Viscoelastic Analysis - Carbon-epoxy $(L / r=3000)$

\begin{tabular}{cccccc}
\hline \hline $\mathrm{G}_{0} / \mathrm{G}_{\infty}$ & $\mathrm{t}_{\infty}^{m}$ & $\mathrm{t}_{\infty}^{l}$ & $\sigma_{f}(\infty)(\mathrm{MPa})$ & $\mathrm{E}_{c}(\infty)(\mathrm{GPa})$ & $\Delta E_{c}(\%)$ \\
\hline \hline 5 & 5.9915 & 8.77 & 362.67 & 178.04 & 1.13 \\
10 & 6.8024 & 12.00 & 361.71 & 177.40 & 1.48 \\
20 & 7.5496 & 28.73 & 360.34 & 176.65 & 1.90 \\
50 & 8.4970 & 85.84 & 357.64 & 175.27 & 2.66 \\
100 & 9.2003 & 243.90 & 354.59 & 173.76 & 3.50 \\
200 & 9.8985 & 766.08 & 350.28 & 171.64 & 4.68 \\
500 & 10.8178 & 1848.20 & 341.73 & 167.45 & 7.01 \\
1000 & 11.5119 & 4768.65 & 332.09 & 162.72 & 9.63 \\
2000 & 12.2056 & 9560.10 & 318.45 & 156.04 & 13.34 \\
\hline \hline
\end{tabular}

$\mathrm{t}_{\infty}^{m}=$ the time required for the matrix shear modulus to stabilize to within $1 \%$ of it's relaxed value

$\mathrm{t}_{\infty}^{\prime}=$ the time required for the system to stabilize, using the linear model

$\Delta E_{c}=$ percentage change in $E_{c}$ due to complete relaxation of the matrix 
AIAA-99-1344

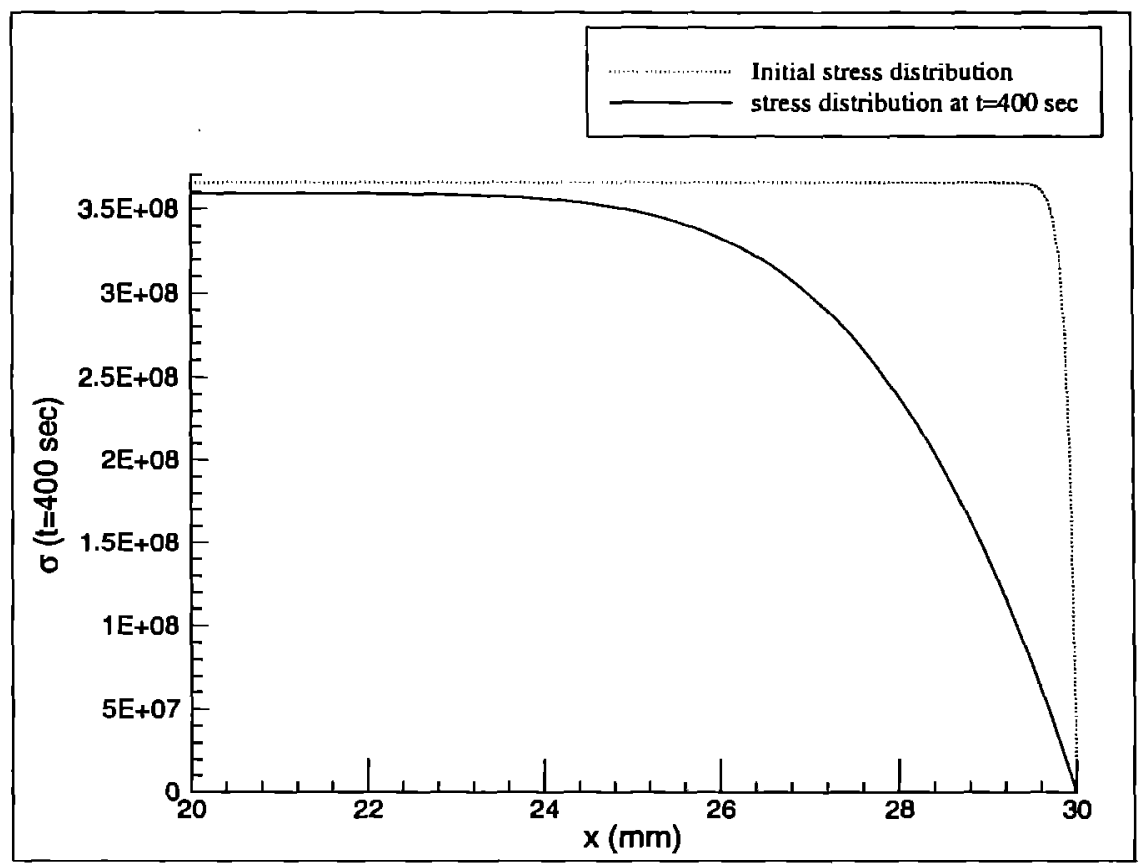

Figure 2: Axial stress distribution from a linear viscoelastic analysis, at times $t=0$ and $t=400 \mathrm{sec}$, Carbon/epoxy, $G_{m}(0) / G_{m}(\infty)=1000$.

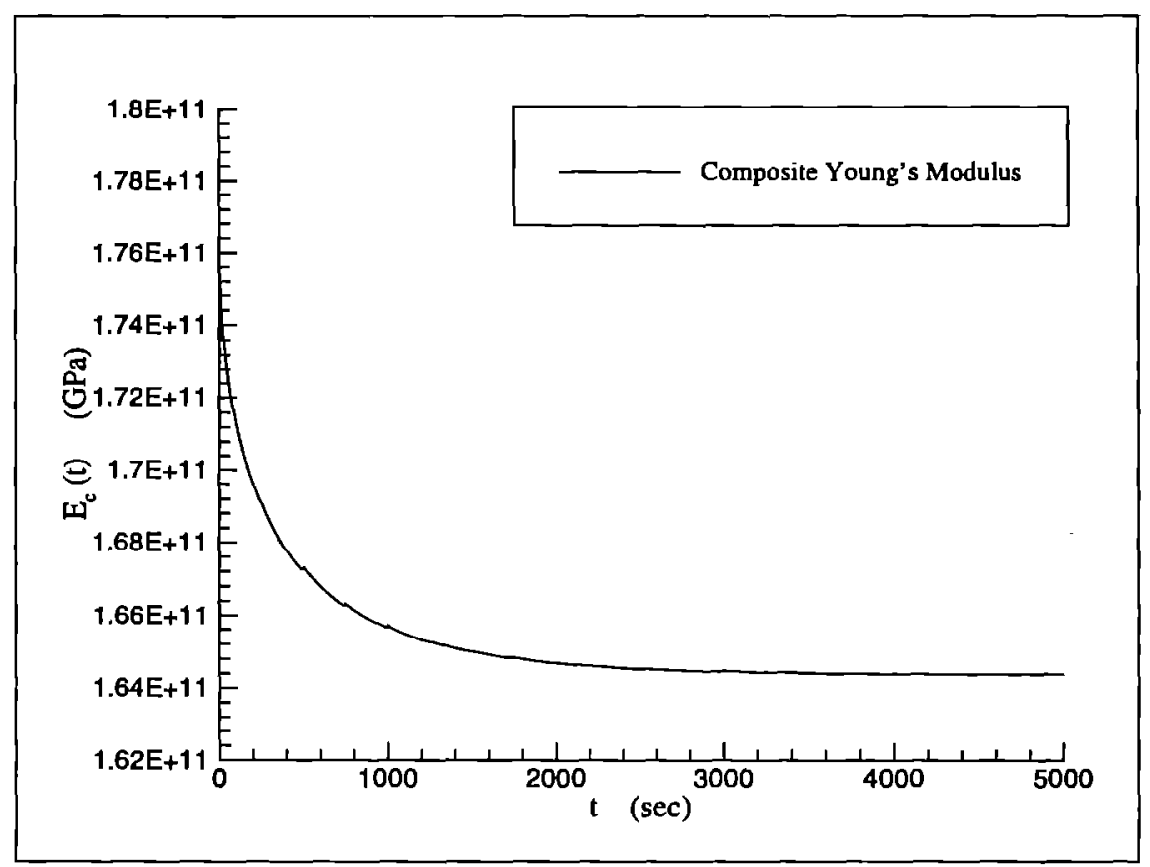

Figure 3: Variation of Composite Axial modulus from a linear viscoelastic analysis, Carbon/epoxy, $G_{m}(0) / G_{m}(\infty)=1000$. 